\title{
Situación emocional de niños y adolescentes que viven con cáncer en Hidalgo
}

\author{
Emotional Situation of Children and Adolescents who live with Cancer in Hidalgo
}

\author{
Sagaón-Olivares AS ${ }^{a}$, Barrera-Gálvez $R^{b}$, Arias-Rico $J^{c}$, Solano-Pérez $C^{d}$, Guevara-Cabrera \\ $R M^{e}$, Baltazar-Téllez $R M^{f}$, Sánchez Padilla $M L^{g}$
}

\begin{abstract}
:
The main theme of this essay is two elements of latent mental health in the ages of children and adolescents: stress and self-esteem; identified when dealing with a condition that requires discretion due to the risks and seriousness that it represents to society: cancer. Known and feared by all, when receiving a news related to cancer, most are amazed with the news. From the context of a stay in a public hospital by children and adolescents receiving chemotherapy as part of their treatment, a non-participant observation was made that led the work team to the following reflection: Are the children and children resilient enough? adolescents how to deal with a cancer situation and the emotionally and physically exhausting treatment it requires? Undoubtedly, it is a constant struggle against the inevitable possibility of premature death, as well as the uncertainty that governs the day to day in the innocence of children, and sometimes, the need for expression of adolescents.
\end{abstract}

Keywords:

Self-esteem, mental load, onco-pediatric

Resumen:

El presente ensayo tiene como tema principal dos elementos de salud mental latentes en edades de niños y adolescentes: estrés y autoestima; identificados al relacionarse con un padecimiento que requiere discreción por los riesgos y gravedad que representa ante la sociedad: el cáncer, sabido y temido por todos, al recibir una noticia relacionada con cáncer, la mayoría se queda asombrado con ella. A partir del contexto de una estancia en hospital público por parte de los niños y adolescentes que reciben quimioterapia como parte de su tratamiento, se realizó una observación no participante que llevó al equipo de trabajo a la siguiente reflexión: ¿son suficientemente resilientes los niños y adolescentes como para afrontar una situación de cáncer y el tratamiento tan desgastante a nivel emocional como físico que requiere? Sin duda, es una lucha constante ante la posibilidad inevitable de una muerte prematura, así como la incertidumbre que gobierna el día a día en la inocencia de los niños, y a veces, en la necesidad de expresión de los adolescentes.

Palabras Clave:

Autoestima, carga mental, onco-pediátrico

\footnotetext{
${ }^{a}$ Universidad Autónoma del Estado de Hidalgo, Instituto de Ciencias de la Salud, ORCID: https://orcid.org/0000-0003-4778-1484, Email: saraisaol15@gmail.com

${ }^{\mathrm{b}}$ Autor de Correspondencia, Universidad Autónoma del Estado de Hidalgo, Instituto de Ciencias de la Salud, ORCID: https://orcid.org/00000002-1949-5424,Email: rosario_barrera@uaeh.edu.mx

${ }^{c}$ Universidad Autónoma del Estado de Hidalgo, Instituto de Ciencias de la Salud, ORCID: https://orcid.org/0000-0003-0219-0410, Email: josearias.rico@hotmail.com

d Universidad Autónoma del Estado de Hidalgo, Instituto de Ciencias de la Salud, ORCID: https://orcid.org/0000-0003-4648-981X, Email: claudia_solano@uaeh.edu.mx

e Universidad Autónoma del Estado de Hidalgo, Instituto de Ciencias de la Salud, ORCID: https://orcid.org/0000-0002-1309-2747, Email: rosy_guevara29@hotmail.com

f Universidad Autónoma del Estado de Hidalgo, Instituto de Ciencias de la Salud, ORCID: https://orcid.org/0000-0002-2168-7564, Email: rbaltazartellez@yahoo.com.mx

g Universidad Autónoma del Estado de Hidalgo, Instituto de Ciencias de la Salud, ORCID: 0000-0003-2628-5932, Email: marialuisasanchezpadilla@yahoo.com.mx
} 


\section{Introducción}

Como es bien sabido y mencionado por los expertos en razonamientos sobre salud en la actualidad: "La salud es el bien más preciado"; y en algunas ocasiones las personas entendemos el valor de la salud hasta que carecemos de ella. Cuando la situación emocional de niños y adolescentes que viven con cáncer es identificada en su estancia en el hospital, pueden surgir diferentes tipos de reacciones, desde las más emotivas con personas abrazando a los niños, pasando por un intermedio de personas que se preocupan y sienten de cierta manera compasión por quienes padecen de este sufrimiento; o simplemente, una observación desde la lejanía, como quien se detiene a mirar un caso, lo estudia, pero no se acerca a ello como si se tratara de una persona, sino solamente un experimento o un número más.

Como parte de los principales problemas de salud pública de nuestra sociedad, encontramos situaciones de estrés y alteraciones emocionales que, en el caso de los niños, no siempre son identificados a tiempo, que no por ello eliminan el riesgo de ser una etapa de las más difíciles en cuanto a emociones se refiere. Por otro lado, la etapa que viven los adolescentes, a veces buscando desafiar o desobedecer y confrontar a sus padres, viene ligada a un "no saber reconocerse" ante la vivencia de un tratamiento de quimioterapia para resolver su alteración física (cáncer).

Sin embargo, hay dos elementos psicológicos importantes ligados a este padecimiento: el estrés con el que se convive para lidiar con la terapia y el padecimiento oncológico, así como la merma en la autoestima de los niños y adolescentes que se enfrentan a esta vivencia, teniendo como principales factores de riesgo: la pérdida de la salud, la amenaza de daño a la propia estructura física, psicológica y social del niño y el adolescente.

\section{Antecedentes}

Las acciones que se puedan emprender con niños y adolescentes en tratamiento oncológico, enfermedades crónicas o en aquellos con enfermedades de difícil curación, son una pieza fundamental para el abordaje de la mejora del bienestar físico, emocional y social, la autoestima, y autonomía de ellos y de sus padres y hermanos, ya que es una problemática que afecta a la familia en su conjunto.

En el ámbito mundial se ha reportado una tendencia en la disminución de la mortalidad por cáncer en niños.
Además, se considera que particularmente las muertes por cáncer en niños y adolescentes han disminuido en países que cuentan con tecnologías terapéuticas más desarrolladas y lo contrario corresponde a los países en desarrollo que no reflejan esta disminución en sus tasas de mortalidad' ${ }^{1}$.

Algunos estudios realizados en Estados Unidos reportan un aumento en la sobrevida de niños con cáncer que han participado en algún protocolo terapéutico. Las tasas de mortalidad disminuyeron de ocho a menos de tres por cada 100,000 niños y la sobrevida aumentó independientemente del tiempo de diagnóstico, a pesar del aumento en la incidencia de la enfermedad. Distintos países de América, Asia, Europa y Oceanía han presentado un patrón muy similar ${ }^{1}$.

En contraste, aún existen países latinoamericanos donde se observa un aumento sostenido de la mortalidad por cáncer en niños y México es uno de los más afectados. Según cifras del Instituto Nacional de Estadística y Geografía (INEGI), en México el cáncer en niños ocupa los primeros lugares en mortalidad y se encuentra sólo por debajo de las muertes accidentales en el grupo de 1 a 14 años. La gran desigualdad que existe en distintas regiones del país, la falta de acceso a servicios de salud y la pobre tecnología con la que se cuenta pueden ser determinantes en el aumento de las tasas de mortalidad en niños, según se ha reportado en la literatura médica internacional ${ }^{1}$.

En términos epidemiológicos, el cáncer pediátrico es la segunda causa de mortalidad en México, siendo la primera causa de muerte por enfermedad. El cáncer infantil se refiere a distintas enfermedades agrupadas en dos ramas de la medicina, por un lado, la oncología se encarga del estudio de los tumores sólidos y por el otro la hematología, quien estudia las alteraciones de la sangre y ambas enfermedades afectan a pacientes pediátricos es decir menores de 18 años. Los pacientes que son diagnosticados por alguna enfermedad de este tipo pueden presentar un impacto psicológico, el cual depende inicialmente de la edad cronológica que tenga.

Una vez iniciado el tratamiento, se pueden presentar cambios físicos como caída del cabello, el aumento o pérdida de peso corporal, cambios de coloración de piel (tonalidad negra en manos, pies y zonas de pliegues), en las uñas (tonalidad café), aparición de múltiples estrías en el abdomen y axilas, hasta la pérdida de la autonomía para las actividades cotidianas como bañarse, vestirse 0 alimentarse, debido a estos aspectos físicos se tiene un gran impacto emocional, que puede alterar su autoestima. 
Las manifestaciones que presentan los niños durante los procedimientos invasivos a los que son sometidos son: agresiones verbales como lenguaje altisonante y agresiones físicas (pellizcos o patadas) dirigidas al personal de salud, y manifestaciones clínicas propias como cambios en las constantes vitales (taquicardia, hipertensión arterial), sudoración, temblores, llanto, enrojecimiento por el esfuerzo físico para evadir el procedimiento, entre otros. Estas alteraciones generan en la persona un incremento en su carga mental, llegando a presentar estrés.

Según datos estadísticos de la UNAM, en México hay más de 5 mil casos de cáncer pediátrico al año; centrándonos al estado de Hidalgo y conforme a datos del Hospital del Niño DIF Hidalgo, en el periodo comprendido en los años 2005 a 2018 se tienen reportados 780 pacientes diagnosticados con cáncer, de los cuales el $46 \%$ pertenece a oncología y el 54\% a hematología, también es relevante y alarmante saber que anualmente se diagnostican 80 casos nuevos de cáncer infantil en dicho hospital.

El impacto psicológico de cada paciente al saber que tiene un diagnóstico de cáncer depende de su edad cronológica, por ejemplo: los lactantes tienen preocupación relacionada con el apego familiar, durante la separación momentánea de sus padres durante la hospitalización, los niños creen ser abandonados por ellos, por otro lado los preescolares manifiestan inquietud por las nuevas rutinas que tendrán que adoptar durante la estancia hospitalaria; los escolares se muestran afligidos porque comprenden un poco más el concepto de la enfermedad y la gravedad, incluso suelen creer que su enfermedad es un castigo por su mal comportamiento, en cuanto a los adolescentes, ellos tienen plena comprensión de la situación que lo aqueja, su angustia está asociada a la gravedad de la enfermedad, las complicaciones del tratamiento y la posible muerte.

La enfermedad se acompaña de pérdida de bienestar físico y psicológico, que alteran la existencia del individuo y su ambiente a corto, mediano y largo plazo. La presencia de una enfermedad, la hospitalización, las cirugías y otros procedimientos médicos son estresores que provocan reacciones psicológicas y biológicas en los pacientes pediátricos $^{2}$.

Todos los niños por si solos son indefensos y especiales, porque se encuentran en proceso de crecimiento y desarrollo, conocimiento y adaptación en la vida; son dependientes de otras personas para realizar diversas actividades, etc. Ahora bien, si a esto le agregamos la presencia de una enfermedad como el cáncer, la vulnerabilidad se agiganta. El curso de la enfermedad traerá en el niño cambios físicos, biológicos, psicológicos entre otros, el impacto y afrontamiento de la enfermedad dependerá inicialmente de la edad cronológica que esté viviendo el niño.

\section{Marco Contextual}

Una de las situaciones que ha merecido la atención de los investigadores desde hace ya algún tiempo ha sido la hospitalización y sus posibles consecuencias estresantes, tanto en la infancia como en adultos. La hospitalización representa una situación donde el sujeto percibe un incremento de su propia vulnerabilidad, al tiempo que se da la presencia de gran cantidad de estímulos estresantes e incluso amenazantes de la propia integridad física ${ }^{3}$.

Conforme a datos del hospital del Niño DIF Hidalgo de los 780 pacientes diagnosticados con cáncer, actualmente se tienen en vigilancia a 340 pacientes, el resto se divide entre recaídas, abandono del tratamiento y defunciones, pero todos ellos tuvieron algo en común, la experiencia de vivir repetidas hospitalizaciones por distintos motivos, como internamiento para ciclos de quimioterapia o por efectos de toxicidad de la misma.

La hospitalización podría provocar en los más pequeños inquietud y ritmos alterados, llantos, problemas con las comidas, conductas regresivas, ansiedad de separación; mientras que en niños de edad preescolar y escolar puede producir tristeza y depresión como síntomas más generalizados. En la adolescencia los miedos más relevantes serían el propio miedo a la estancia hospitalaria, el miedo a los procedimientos médicos (inyecciones, dolor, entre otros) y el miedo a la enfermedad y sus posibles repercusiones ${ }^{3}$.

La duración de la estancia en el hospital podría tener una gran importancia, una hospitalización media de más de una semana o repetidas readmisiones de pequeña duración podrían asociarse con un considerable aumento de las alteraciones conductuales del paciente pediátrico y con un aumento de su estrés ${ }^{3}$.

El tiempo aproximado para el tratamiento de los padecimientos hematológicos es de 145 semanas y de 6 meses para los oncológicos ${ }^{4}$; sin incluir las frecuentes hospitalizaciones por complicaciones agregadas, aunado a esto durante el tratamiento estos pacientes son sometidos a múltiples procedimientos médicos invasivos y dolorosos. Los pacientes oncológicos están expuestos a factores estresantes continuos, pues el tiempo de hospitalización para el ciclo de quimioterapia es de 3 a 7 
días, pero si el motivo de ingreso al hospital es por toxicidad quimioterapéutica los días son de 10 a 14, incluso hasta 21 si se cursa con una infección grave.

Enfocándonos a los pacientes en tratamiento quimioterapéutico consultamos el estudio de Carvalho Leite "Evaluación de la autoestima en pacientes oncológicos sometidos a tratamiento quimioterápico" donde refiere que durante el tratamiento del cáncer el paciente puede hacer frente a posibles cambios en la apariencia física, limitaciones e impedimentos de actividades rutinarias que a menudo añadieron a la quimioterapia, al estigma de la enfermedad, a la dificultad para enfrentar el tratamiento y sus efectos secundarios, y a la rehabilitación después del tratamiento. Con eso, estos pacientes pueden tener problemas psicológicos, como cambios en su autoestima, una vez que su percepción de la imagen corporal está relacionada con esta nueva condición de vida ${ }^{5}$.

\section{Autoestima}

Se entiende como autoestima a la capacidad que tiene la persona de valorarse, amarse, apreciarse y aceptarse a sí mismo ${ }^{6}$.

Hablando sobre el periodo de tratamiento quimioterapéutico la autoestima de los niños y adolescentes está relacionada con muchas formas de conducta, como menciona Muñoz Campos en su análisis de la autoestima, las personas con una autoestima elevada suelen reportar menos emociones agresivas, negativas y menos depresión que las personas con una autoestima baja. Las personas con una autoestima elevada pueden manejar mejor el estrés y cuando son expuestas al mismo, experimentan menos efectos negativos en la salud ${ }^{6}$.

De tal forma que, el paciente oncológico al tener una autoestima alta, desencadena pensamientos positivos de sí mismo y el entorno hospitalario que lo rodea, disminuye el estrés, y tiene una adecuada disposición para mejor la salud y como resultado se tiene un idóneo apego al tratamiento.

Por otro lado, Nathaniel Branden, psicoterapeuta canadiense, en su libro "Los seis pilares de la autoestima" dice que la autoestima, plenamente consumada, es la experiencia fundamental de que podemos llevar una vida significativa y cumplir sus exigencias ${ }^{7}$. Hemos observado pacientes oncológicos adolescentes que durante la hospitalización para el ciclo de quimioterapia realizan actividades como la elaboración de llaveros de madera pintados por ellos mismo, bordado de servilletas, tejido de bufandas, entre otros, para posteriormente comercializaros, lo cual no solo los ayuda de manera económica, sino emocionalmente, pues los hace sentir personas productivas dentro de la sociedad, manteniendo la certeza de que se puede tener una vida significativa a pesar la enfermedad.

Por otra parte, también se puede estudiar la autoestima desde dos elementos importantes como lo hizo BarreraGalvéz autora el estudio "Desarrollo de Capacidades Afectivas en Estudiantes de Enfermería en la UAEH" quien considera que la autoestima es confiar en las propias potencialidades y menciona dos componentes: la valía personal y el sentimiento de capacidad personal. La primera se refiere a la valoración positiva o negativa que la persona tiene de su auto concepto, incluyendo las actitudes hacia sí mismo; la segunda alude a las expectativas que tiene una persona de ser capaz, de hacer de manera exitosa lo que tiene que hacer, es decir, su autoeficacia ${ }^{8}$.

El paciente oncológico posee una vulnerabilidad doble, por lo que consideramos relevante el estudio de sus emociones durante la fase de tratamiento hospitalario para poder identificar manifestaciones evidentes de alteraciones en su autoestima.

Dentro del entorno hospitalario podemos observar adolescentes hostiles con comportamientos de aislamiento social, es decir, ellos se limitan únicamente a interactuar de manera esporádica con el personal de salud, igualmente con sus propios padres, inclusive en ocasiones suelen cubrirse el rostro o el cuerpo completo con una sábana, también, se niegan a realizar actividades de higiene, cambio de bata, incluso no permiten el cambio de las sábanas, ni la realización de procedimientos básicos como medición de signos vitales y la toma de medicamentos vía oral, les es indiferente su arreglo personal 0 las actividades recreativas intrahospitalarias pues no les generan motivación alguna. Por otro lado, el constante enojo, las agresiones físicas y los pensamientos de minusvalía están presentes en los escolares.

\section{Carga Mental}

La carga mental es el conjunto de requerimientos mentales, cognitivos o intelectuales a los que se ve sometido una persona durante la realización de una tarea ${ }^{9} \mathrm{o}$ bien durante una actividad establecida.

La carga mental se identifica como el estrés el cual ha sido desde tiempo atrás tema relevante de estudio, debido a esto existen distintas formadas de medirlo, 
desde la aplicación de un sencillo cuestionario hasta la compleja utilización de dispositivos tecnológicos que determinan su presencia. Sandra Hart investigadora de la Administración de Aeronáutica y del Espacio (NASA) diseño el "Método NASA-TLX Task Load Index" que supone un procedimiento de valoración de la carga mental desde una perspectiva multidimensional (exigencias: mentales, físicas, temporales y de rendimiento, esfuerzo y nivel de frustración) ${ }^{10}$ este método de tal manera que pueda ser utilizado para evaluar a cualquier persona que se encuentre bajo una situación de estrés, sin importar el área en donde se encuentra, puede ser laboral, académica, e incluso hospitalaria.

síntomas momentáneos como: ansiedad, temor, irritabilidad, tristeza hasta llegar al punto de la somatización, presentando dolor de cabeza, estomago, dificultad para respirar, dermatitis, entre otros. Incluso podría también aumentar la gravedad del cuadro clínico de una enfermedad en curso o bien impedir el normal funcionamiento del cuerpo humano.

El estrés desde hace tiempo a sigo objeto de estudio desde múltiples variables para muchos investigadores, al revisar la literatura sobre el estrés asociado del curso de enfermedades crónicas encontramos que Julieta Garay López en su estudio "Acontecimientos productores del estrés en pacientes oncológicos" sugiere que los efectos del estrés dependen de la percepción del estresor, la capacidad del individuo para controlar una situación estresante así como su preparación en su sociedad para afrontar problemas y la influencia de los patrones de conducta aprobados por esta sociedad. Arguye que el estrés afecta a la salud dependiendo de los recursos personales y de la personalidad, así como también de los recursos sociales ${ }^{12}$.

\section{Relación Enfermera- Paciente}

Durante la práctica diaria las enfermeras del servicio de oncología y quimioterapia ambulatoria, son quienes observan detalladamente las manifestaciones que viven los niños durante los constantes procedimientos invasivos a los que son sometidos tras el tratamiento del cáncer, a pesar de estar casi en todo momento en compañía de sus padres, los pacientes presentan reacciones por niveles de estrés altos, la mayoría generados por pensamientos asociadas al dolor, por ejemplo: las punciones durante la medición de glicemia capilar, toma de muestra sanguínea para estudio de laboratorio, colocación de accesos vasculares (catéteres), instalación de aguja para catéter puerto; y son detonadas cuando ven directa y cercanamente los punzocortantes, algunas de manifestaciones son:
La Organización Mundial de la Salud (OMS) a partir de 1994 define al fenómeno del estrés como las reacciones fisiológicas que en su conjunto preparan al organismo para la acción. Es decir, el estrés sería una alarma, un estímulo que conduce a la acción, una respuesta necesaria para la supervivencia, respuesta que puede ser coherente con las demandas del entorno, o bien, insuficientes 0 exageradas ${ }^{11}$.

Se ha observado en diferentes investigaciones que el cáncer está asociado al estrés psicológico ${ }^{12}$, el acúmulo de estrés en cualquier individuo puede originar desde aparición de simples

agresiones verbales como lenguaje altisonante y agresiones físicas (pellizcos, patadas, expectoraciones) dirigidas al personal de salud, y manifestaciones clínicas propias como cambios en las constantes vitales (taquicardia, hipertensión arterial), sudoración, temblores, llanto, enrojecimiento por el esfuerzo físico para evadir el procedimiento, entre otros.

Hablando un poco sobre el gremio de Enfermería, es relevante recordar el importante rol que tiene la enfermera dentro del equipo interdisciplinario de salud, pues la terapéutica que proporciona requiere que ella permanezca directamente con el paciente las 24 horas del día, bridando cuidados holísticos que promueven la calidad de vida; la relación interpersonal enfermerapaciente es la clave básica para la realización de las actividades e intervenciones, de esta manera se facilita la adherencia al tratamiento, como resultado de ello, la familiaridad que se genera durante el contacto del niño con su enfermera, permite que el paciente sienta la confianza de expresar sus emociones durante el largo transcurso que conlleva un tratamiento oncológico.

\section{Conclusión}

Los niños son vulnerables, por lo que requieren de ser cuidados y protegidos principalmente por sus padres y su entorno familiar; si durante la infancia se cursa con una enfermedad como el cáncer, se vuelven aún más vulnerables; el impacto personal y el afrontamiento de la enfermedad dependerá de múltiples variables, como: a) las personales, que son aquellos que dependen únicamente del niño; b) las familiares, que pueden incluir el apego del niño con sus padres y hermanos; c) las hospitalarias, que engloban el contacto y el ambiente al que se enfrenta el paciente durante la hospitalización; sin embargo, las tres tiene un impacto a nivel emocional que genera alteraciones en la autoestima y niveles altos de estrés en el paciente oncológico. Una de las estrategias que hemos visto en estos procesos es el importante papel 
de una enfermera con el paciente con cáncer infantil donde favorece y fortalece la parte asistencial en los hospitales.

\section{Referencias}

[1] Escamilla-Santiago RA, Narro-Robles J, Fajardo-Gutierrez A, Rascón Pacheco RA, López-Cervantes M. (2012) Tendencias de la mortalidad por cáncer en niños y adolescentes según grado de marginación en México (1990-2009). Salud Pública de Mex. Vol. 54. No. 6.

[2] Ortigosa-Quiles JM, Méndez FX, Pedroche S. (1999) Cuestionario de preocupaciones sobre cirugía infantil Psicothema. Vol. 11 (3): 601-609.

[3] Fernández Castillo A, López Naranjo I. (2006) Transmisión de emociones, miedo y estrés infantil por hospitalización. Int.J.Clinic.Health.Psych. Vol. 6 (3) 631-645.

[4] Rivera Luna R. (2007) El niño con cáncer: los padecimientos más comunes para el médico no especialista. 1a ed. Editores de textos mexicanos. México.

[5] Carvalho Leite M A, Alves Nogueira D, De Sousa Terra F. (2015) Evaluación de la autoestima en paciente oncológicos sometidos a tratamiento quimioterápico. Rev.latino-am.Enfermagen. Vol. 23(6): 1082-9.

[6] Muñoz/Campos. (1992) Lidezgo y mercadeo. Autoestima. Publicación de la Universidad de Guanajuato. México.

[7] Branden N. (1995) Los seis pilares de la autoestima. $1^{\mathrm{a}}$ ed. Paidos Ibérica; España.

[8] Barrera-Gálvez R, Solano-Pérez CT. Arias-Rico J, et al. (2018) "Desarrollo de Capacidades Afectivas en Estudiantes de Enfermería en la UAEH". Salud y Educación. Boletín Vol. 7(3).

[9] Sebastian García O, Del Hoyo Delgado Ma. A. (2002) La carga mental del trabajo-INSHT: España.

[10]Barrera Gálvez R. Díaz Pérez LE. Busto Villarreal JM, Romero Quezada LC, Domínguez Ramírez OA. (2014) Realización de una evaluación de un sistema de interacción física hombre-robot con base en el protocolo NASA-TLX. UAEH. No.5 (3).

[11]OMS (2015) El estrés según la Organización Mundial de la Salud. Suiza.

[12]Garay López J, Moysen Chimal A, Esteban Valdés J M, Balázar Nava P, Gurrola Peña G M. (2012) Acontecimientos productores del estrés en pacientes oncologicos. Rev. Psicología: nueva época. UAEMex. Vol1.no.2 pp 46-57. 